\title{
THE ONSET OF DOUBLE-DIFFUSIVE CONVECTION IN A LAYER OF NANOFLUID UNDER ROTATION
}

\author{
G. C. Rana ${ }^{\mathrm{a}}$, \\ and R. C. Thakur \\ ${ }^{\text {a }}$ Department of Mathematics, NSCBM \\ Government College, Hamirpur - 177005 \\ Himachal Pradesh, INDIA \\ drgcrana15@gmail.com \\ ${ }^{\mathrm{b}}$ Department of Mathematics, Govt. College \\ Nurpur, District Kangra \\ Himachal Pradesh, INDIA \\ rameshnahan@yahoo.com \\ Received: April 25, 2016 \\ Revised: May 23, 2016 \\ Accepted: June 01,2 016

\section{ABSTRACT} \\ Double-diffusive convection in a horizontal layer of nanofluid under \\ rotation heated from below is studied. The nanofluid describes the \\ effects of thermophoresis and Brownian diffusion. Based upon \\ perturbations and linear stability theory, the normal mode analysis \\ method is applied to obtain the dispersion relation characterizing the \\ effect of different parameters when both the boundaries are free. Due to \\ thermal expansion, the nanofluid at the bottom will be lighter than the \\ fluid at the top. Thus, this is a top heavy arrangement which is \\ potentially unstable. In this paper we discuss the influences of various \\ non-dimensional parameters such as rotation, solute gradient, thermo- \\ nanofluid Lewis number, thermo-solutal Lewis number, Soret and \\ Dufour parameter on the stability of stationary convection for the case \\ of free-free boundaries. It is observed that rotation and solute gradient \\ have stabilizing influence on the system. Rotation and solute gradient \\ play important role in the thermal convection of fluid layer and has \\ applications in rotating machineries such as nuclear reactors, petroleum \\ industry, biomechanics etc. and solute gradient finds applications in \\ geophysics, food processing, soil sciences, oil reservoir modeling, \\ oceanography etc. A very good agreement is found between the present \\ paper and earlier published results. \\ Keywords: convection, nanofluid, nanoparticles, rotation, soret and dufour \\ parameter
}

\section{NOMENCLATURE}

a Wave number

c Specific heat

d Thickness of the horizontal layer

$\mathrm{D}_{\mathrm{B}} \quad$ Brownian diffusion coefficient $\left(\mathrm{m}^{2} / \mathrm{s}\right)$

$\mathrm{D}_{\mathrm{T}} \quad$ Thermophoretic diffusion coefficient

$\mathrm{G} \quad$ Acceleration due to gravity $\left(\mathrm{m} / \mathrm{s}^{2}\right)$

g Gravitational acceleration vector

$\mathrm{k} \quad$ Thermal conductivity of nanofluid $(\mathrm{W} / \mathrm{mK})$

Le Thermosolutal Lewis number

Ln Thermo-nanofluid Lewis number

$\mathrm{N}_{\mathrm{A}} \quad$ Modified diffusivity ratio

$\mathrm{N}_{\mathrm{B}} \quad$ Modified particle-density ratio

$N_{\text {CT }} \quad$ Soret parameter

$N_{\text {TC }} \quad$ Dufour parameter

$\mathrm{p} \quad$ Pressure $(\mathrm{Pa})$

q Darcy velocity vector $(\mathrm{m} / \mathrm{s})$

$R_{D} \quad$ Thermal Darcy-Rayleigh number

$\left(R_{D}\right)_{C}$ Critical Thermal Darcy-Rayleigh number

$\mathrm{Rm} \quad$ Basic-density Rayleigh number

$\mathrm{Rn} \quad$ Concentration Rayleigh number

Rs Solutal Rayleigh number

$\mathrm{t}$ time (s)

$\mathrm{T}$ temperature (K)

Ta Taylor Number

(u,v,w) Darcy velocity components

$(\mathrm{x}, \mathrm{y}, \mathrm{z})$ space co-ordinates $(\mathrm{m})$

\section{Greek symbols}

$\begin{array}{ll}\alpha_{T} & \text { Solute volumetric coefficient } \\ \alpha_{C} & \text { Thermal volumetric coefficient }(1 / \mathrm{K}) \\ \varphi & \text { Nanoparticles volume fraction } \\ \mu & \text { Viscosity of the fluid }\left(\mathrm{Ns} / \mathrm{m}^{2}\right) \\ \rho & \text { Density of fluid }\left(\mathrm{kg} / \mathrm{m}^{3}\right) \\ \rho_{p} & \text { Nanoparticle mass density }\left(\mathrm{kg} / \mathrm{m}^{3}\right) \\ \omega & \text { Growth rate of disturbances }\end{array}$

\section{Superscripts}

' Non-dimensional variables

' ' Perturbed quantity

\section{Subscripts}

p Particle

f Fluid

b Basic state

$0 \quad$ Lower boundary

1 Upper boundary

\section{INTRODUCTION}

It Double-diffusive convection is an important field of research for the last few decades that has applications in various branches such as geophysics, 
soil sciences, food processing, oil reservoir modeling, oceanography, limnology and engineering etc. Chandrasekhar (19610 has discussed in details the thermal instability of a Newtonian fluid under the various assumptions of hydrodynamics and hydromagnetics. Double-diffusive convection problems related to different types of fluids and geometric configurations have been extensively studied by Brakke (1955), Stomel and Fedrov (1967), Turner (1974), Sharma et al. (2001) and Rana et al. (2012). Rotation plays an important role in the thermal convection of fluid layer and has applications in rotating machineries such as nuclear reactors, petroleum industry, bio mechanics etc.

In recent years, most attention of researchers is to study the problems of thermal convection in nanofluids having relevance in several industries such as the automotive, pharmaceutical or energy supply industries. The term nanofluid was first coined by Choi 91995). A nanofluid is a colloidal suspension of nano sized particles, that is, particles the size of which is below $100 \mathrm{~nm}$, in a base fluid. Common fluids such as water, ethanol or engine oils are typically used as base fluids in nanofluids. Due to enhanced thermal conductivity of nanofluids being used as great coolants and suspensions of nanoparticles are being developed medical applications including cancer therapy. The absolute nanoparticle velocity can be viewed as the sum of the base fluid velocity and a relative slip velocity as discussed by Buongiorno (2006). After analyzing the effect of the following seven slips mechanisms: inertia, Brownian diffusion, thermophoresism, diffusiophoresis, Magnus effect, Fluid drainage and gravity, he concluded that in the absence of turbulent eddies Brownian diffusion and thermophoresis are the dominant slip mechanisms. Thus, convection in nanofluids based on Buongiorno's model has attracted great interest.

Thermal convection problems in a horizontal layer saturated by a nanofluid have also been discussed by Tzou (2008), Alloui et al. (2011), Nield and Kuznetsov (2009), Kuznetsov and Nield (2010a, b) and Chand and Rana (2012a). Rotation plays important role in the thermal instability of fluid layer and has applications in rotating machineries such as nuclear reactors, petroleum industry, biomechanics etc. Owing to their enhanced properties as thermal transfer fluids for instance, nanofluids can be used in a plethora of engineering applications ranging from use in the automotive industry to the medical arena to use in power plant cooling systems as well as computers.

Nield and Kuznetsov (2011) studied the doublediffusive convection in a layer of nanofluids while the effect of rotation on thermal convection in the nanofluid layer saturating a Darcy-Brinkman porous medium studied by Chand and Rana (2012b) and found that rotation has stabilizing effect on the system. Rana (2013) studied the thermsolutal convection in Walters' (Model B') rotating fluid permeated with suspended particles and variable gravity field in porous medium in hydromagnetics and found that rotation has stabilizing effect on stationary convection of the system when both the boundaries are free while Rana et al. (2014) studied the Dufour and Soret effects on double-diffusive instability of non-Newtonian fluid in porous medium and found that Dufour parameter has destabilizing effect and Soret parameter has stabilizing effect on the onset of stationary convection.

Keeping in view of various applications as mentioned above, the effect of rotation on doublediffusive convection in a layer of nanofluid is studied in this paper. This paper is the extension of paper studied by Nield and Kuznetsov (2011) which includes one additional term namely, rotation parameter and also the extension of the paper studied by Chand and Rana (2012b), which includes three additional terms namely, solute gradient, Soret and Dufour parameters.

\section{MATHEMATICAL MODEL}

Consider an infinite horizontal layer of nanofluid of thickness $d$, bounded by the planes $\mathrm{z}=$ 0 and $z=d$. The layer is heated and soluted from below, which is acted upon by a gravity force $g=(0$, $0,-g$ ) aligned in the $z$ direction and rotating uniformly about $\mathrm{z}$-axis with angular velocity $\Omega(0$, $0, \Omega$ ) as shown in fig.1. Each boundary wall is assumed to be impermeable and perfectly thermal conducting. The temperature $T$, concentration $C$ and the volumetric fraction of nanoparticles $\varphi$ at the lower (upper) boundary is assumed to take constant values $T_{0}, C_{0}$ and $\varphi_{0}\left(T_{1}, C_{1}\right.$ and $\left.\varphi_{1}\right)$, respectively.

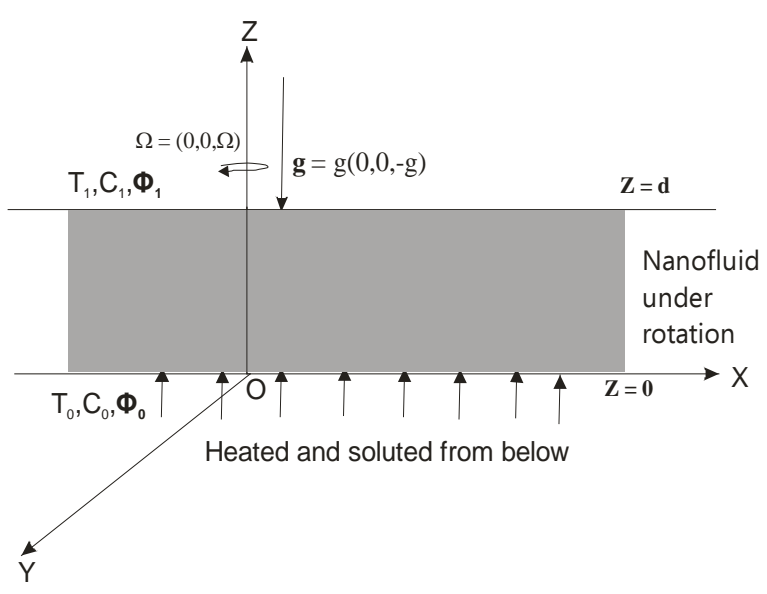

Figure 1. Schematic sketch of physical situation.

\section{BASIC ASSUMPTIONS}

The physical model of the problem is based upon the following assumptions:

- All thermo physical properties, except for the density in the buoyancy term, are constant 
(Boussinesq approximation)

- Base fluid and nano particles are in thermal equilibrium state;

- Nanofluid is incompressible and laminar;

- Negligible radiative heat transfer

- The temperature, the solute concentration and the volumetric fraction of the nanoparticles are constant on the boundaries.

- Nanoparticles do not affect the solute concentration.

\section{FORMULATION OF THE PROBLEM}

Let $\rho, \mu, p$ and $\mathbf{q}(\mathrm{u}, \quad \mathrm{v}, \mathrm{w})$, denote respectively, the density, viscosity, pressure, and Darcy velocity vector. Then the equations of massbalance and momentum-balance for nanofluid [Chandrasekhar (1962), Kuznetsov and Nield (2010 b), Rana et al. (2012), Chand and Rana (2012a, b)] under rotation are

$$
\begin{gathered}
\nabla \cdot \mathbf{q}=0, \\
\rho \frac{d \mathbf{q}}{d t}=-\nabla p+\mu \nabla^{2} \mathbf{q}+\frac{2 \rho}{\varepsilon}(\mathbf{q} \times \mathbf{\Omega}) \\
+\left(\begin{array}{l}
\varphi \rho_{p}+ \\
(1-\varphi)\left\{\rho_{f}\left(1-\alpha_{T}\left(T-T_{0}\right)-\alpha_{C}\left(C-C_{0}\right)\right)\right\}
\end{array}\right),
\end{gathered}
$$

where $\frac{d}{d t}=\frac{\partial}{\partial t}+(\mathbf{q} \cdot \nabla)$ is the convective derivative, $\varphi$ is the volume fraction of nanoparticles, $\rho_{p}$ is the density of nanoparticles and $\rho_{f}$ is the density of base fluid, $\alpha_{T}$ is the coefficient of thermal expansion and $\alpha_{C}$ is analogous to solute concentration and we approximate the density of the nanofluid by that of the base fluid that is we consider $\rho=\rho_{f}$ [Boungiorno (2006), Tzou (2008) and Kuznetsov and Nield (2010a, b).

The equation of mass-balance for the nanoparticles [Buongiorno (2006)] is

$$
\frac{\partial \varphi}{\partial t}+\mathbf{q} \cdot \nabla \varphi=D_{B} \nabla^{2} \varphi+\frac{D_{T}}{T_{1}} \nabla^{2} T
$$

The thermal energy-balance equation for a nanofluid is

$$
\begin{aligned}
& (\rho \mathrm{c})_{\mathrm{f}}\left[\frac{\partial \mathrm{T}}{\partial \mathrm{t}}+\mathbf{q} \cdot \nabla \mathrm{T}\right]=\mathrm{k}_{\mathrm{m}} \nabla^{2} \mathrm{~T}+ \\
& (\rho \mathrm{c})_{\mathrm{p}}\left(\mathrm{D}_{\mathrm{B}} \nabla \varphi \cdot \nabla \mathrm{T}+\frac{\mathrm{D}_{\mathrm{T}}}{\mathrm{T}} \nabla \mathrm{T} . \nabla \mathrm{T}\right)+\rho c D_{T C} \nabla^{2} C,
\end{aligned}
$$

where $(\rho c)_{f}$ is heat capacity of fluid, $(\rho c)_{p}$ is heat capacity of nano particles and $k_{m}$ is thermal conductivity and $D_{T C}$ is a diffusivity of Dufour type. The conservation equation for solute concentration [Kuznetsov and Nield (2010a)] is

$\frac{\partial C}{\partial t}+\mathbf{q} \cdot \nabla C=D_{S m} \nabla^{2} C+D_{C T} \nabla^{2} T$

where $D_{S m}$ and $D_{C T}$ are respectively, the solute diffusivity and diffusivity of Soret type (commonly a Soret parameter $S$ defined by $S=\frac{\alpha_{c} D_{C T}}{\alpha_{T} D_{S m}}$ is employed in lieu of $D_{C T}$ ).

The boundary conditions relevant to the problem are

$$
\begin{gathered}
\mathrm{w}=0, \quad \mathrm{~T}=\mathrm{T}_{0}, \quad \varphi=\varphi_{0}, C=\mathrm{C}_{0} \text { at } \quad \mathrm{z}=0, \\
\mathrm{w}=0, \quad \mathrm{~T}=\mathrm{T}_{1}, \quad \mathrm{C}=\mathrm{C}_{1} \quad \varphi=\varphi_{1} \text { at } \quad \mathrm{z}=1 .
\end{gathered}
$$

Introduce non-dimensional variables

$$
\begin{aligned}
& \left(\mathrm{x}^{\prime}, \mathrm{y}^{\prime}, \mathrm{z}^{\prime},\right)=\left(\frac{\mathrm{x}, \mathrm{y}, \mathrm{z}}{\mathrm{d}}\right), \\
& \left(\mathrm{u}^{\prime}, \mathrm{v}^{\prime}, \mathrm{w}^{\prime},\right)=\left(\frac{\mathrm{u}, \mathrm{v}, \mathrm{w}}{\kappa_{\mathrm{m}}}\right) \mathrm{d}, \mathrm{t}^{\prime}=\frac{\mathrm{t \kappa}}{\sigma \mathrm{d}^{2}}, \\
& \mathrm{p}^{\prime}=\frac{\mathrm{pk}}{\mu \kappa_{\mathrm{m}}}, \varphi^{\prime}=\frac{\left(\varphi-\varphi_{0}\right)}{\left(\varphi_{1}-\varphi_{0}\right)}, \\
& \mathrm{T}^{\prime}=\frac{\left(\mathrm{T}-\mathrm{T}_{1}\right)}{\left(\mathrm{T}_{0}-\mathrm{T}_{1}\right)}, \mathrm{C}^{\prime}=\frac{\left(\mathrm{C}-C_{1}\right)}{\left(\mathrm{C}_{0}-C_{1}\right)},
\end{aligned}
$$

where $\kappa_{\mathrm{m}}=\frac{\mathrm{k}_{\mathrm{m}}}{\left(\rho \mathrm{c}_{\mathrm{p}}\right)_{\mathrm{f}}}$ is thermal diffusivity of the fluid.

Dropping the dashes ( ' ) for convenience.

In non-dimensional form, Eqs. (1) -(5) can be written as

$$
\nabla \cdot \mathbf{q}=0
$$

$$
\begin{gathered}
\frac{1}{\operatorname{Pr}}\left[\frac{\partial \mathbf{q}}{\partial t}+\mathbf{q} \cdot \nabla \mathbf{q}\right]=-\nabla p+\nabla^{2} \mathbf{q}-R m \hat{e}_{z} \\
+\operatorname{RaT} \hat{e}_{z}-\operatorname{Rn} \varphi \hat{e}_{z}+\sqrt{T a}\left(q \times \hat{e}_{z}\right), \\
\frac{\partial \varphi}{\partial t}+\mathbf{q} \cdot \nabla \varphi=\frac{1}{L n} \nabla^{2} \varphi+\frac{N_{A}}{L n} \nabla^{2} T,
\end{gathered}
$$




$$
\begin{aligned}
& \frac{\partial T}{\partial t}+\mathbf{q} \cdot \nabla T=\nabla^{2} T+\frac{N_{B}}{L n} \nabla \varphi \cdot \nabla T \\
& +\frac{N_{A} N_{B}}{L n} \nabla T \cdot \nabla T+N_{T C} \nabla^{2} C, \\
& \frac{\partial C}{\partial t}+\mathbf{q} \cdot \nabla C=\frac{1}{L e} \nabla^{2} \varphi+N_{C T} \nabla^{2} T,
\end{aligned}
$$

where we have dimensionless parameters:

$\operatorname{Pr}=\frac{\mu}{\rho \kappa_{m}} ; \quad$ is the Prandtl number,

$L e=\frac{\kappa_{m}}{D_{S}} ;$ is the thermo-solutal Lewis number,

$\operatorname{Ln}=\frac{\kappa_{m}}{D_{B}}$; is the thermo-nanofluid Lewis

number,

$T a=\left(\frac{2 \Omega d^{2}}{v}\right)^{2} ;$ is the modified Taylor

number

$R_{D}=\frac{\operatorname{\rho g} \alpha \mathrm{d}\left(T_{0}-T_{1}\right)}{\mu \kappa_{m}} ;$ is the thermal

Rayleigh-Darcy Number

Solutal Rayleigh Number

$R s=\frac{\operatorname{\rho g} \alpha \mathrm{d}\left(C_{0}-C_{1}\right)}{\mu D_{S_{m}}}$;

Density Rayleigh number

$\mathrm{R}_{\mathrm{m}}=\frac{\rho_{\mathrm{p}} \varphi_{0}+\rho\left(1-\varphi_{0}\right) \mathrm{gd}}{\mu \kappa_{\mathrm{m}}}$;

Nanoparticle Rayleigh number

$\mathrm{Rn}=\frac{\left(\rho_{\mathrm{p}}-\rho\right)\left(\varphi_{1}-\varphi_{0}\right) \mathrm{gd}}{\mu \kappa_{\mathrm{m}}} ;$

Modified diffusivity ratio

$N_{A}=\frac{D_{T}\left(T_{0}-T_{1}\right)}{D_{B} T_{1}\left(\varphi_{1}-\varphi_{0}\right)}$;

Modified particle- density ratio

$\mathrm{N}_{\mathrm{B}}=\frac{(\rho \mathrm{c})_{\mathrm{p}}\left(\varphi_{1}-\varphi_{0}\right)}{(\rho \mathrm{c})_{\mathrm{f}}}$;
Soret parameter $N_{T C}=\frac{D_{T C}\left(C_{0}-C_{1}\right)}{\kappa_{m}\left(T_{0}-T_{1}\right)}$;

The dimensionless boundary conditions are

Dufour parameter $N_{C T}=\frac{D_{C T}\left(T_{0}-T_{1}\right)}{\kappa_{m}\left(C_{0}-C_{1}\right)}$;

$w=0, \quad \mathrm{~T}=1, \mathrm{C}=1, \varphi=0$ at $\mathrm{z}=0$,

$w=0, T=0, C=0, \quad \varphi=1 \quad$ at $z=1$.

\section{BASIC SOLUTIONS}

We assume a quiescent basic state by following Kuznetsov and Nield (2010a, b) and Chand and Rana (2012b) that verifies

$$
\begin{aligned}
& u=v=w=0, p=p_{b}(z), C=C_{b}(z), \\
& T=T_{b}(z), \varphi=\varphi_{b}(z) .
\end{aligned}
$$

Substituting the basic state defined in (27) into Eqs. (9) - (13), we get

$$
\begin{gathered}
0=-\frac{d p_{b}(z)}{d z}-R m+R_{D} T_{b}(z)+\frac{R s}{L e} C_{b}(z) \\
-R n \varphi_{b}(z), \\
\frac{d^{2} \varphi_{b}(z)}{d z^{2}}+N_{A} \frac{d^{2} T_{b}(z)}{d z^{2}}=0,
\end{gathered}
$$

$$
\begin{gathered}
\frac{d^{2} T_{b}(z)}{d z^{2}}+\frac{N_{B}}{L n} \frac{d \varphi_{b}(z)}{d z} \frac{d T_{b}(z)}{d z} \\
+\frac{N_{A} N_{B}}{L e}\left(\frac{d T_{b}(z)}{d z}\right)^{2}+N_{\text {TC }} \frac{d^{2} C_{b}(z)}{d z^{2}}=0, \\
\frac{1 d^{2} C_{b}(z)}{L e}+N_{C T} \frac{d^{2} T_{b}(z)}{d z^{2}}=0,
\end{gathered}
$$

Solving Eq. (29) by using boundary conditions (25) and (26), we obtain

$$
\varphi_{b}(z)=\left(1-T_{b}\right) N_{A}+\left(1-N_{A}\right) z .
$$

Solving Eq. (31) by using boundary conditions (25) and (26), we obtain

$$
C_{b}(z)=\left(1-T_{b}\right) L e N_{C T}-\left(1+L e N_{C T}\right) z+1 .
$$


Using Eqs. (32) and (33) in Eq. (30), we get

$$
\frac{d^{2} T_{b}(z)}{d z^{2}}+\frac{\left(1-N_{A}\right) N_{B}}{\operatorname{Ln}\left(1+N_{T C} N_{C T} L e\right)} \frac{d T_{b}(z)}{d z}=0 .
$$

Solving the differential Eq. (34) by using boundary conditions (25) and (26), we obtain

$T_{b}(z)=\frac{1-e^{-\left(1-N_{A}\right) N_{B}(1-z) / L n\left(1+N_{T C} N_{C T} L e\right)}}{1-e^{-\left(1-N_{A}\right) N_{B} / L n\left(1+N_{T C} N_{C T} L e\right)}}$

For most nanofluid investigated so far $L_{n} /\left(\varphi-\varphi_{0}\right)$ is large [Buongiorno (2 006 )] of order $10^{5}-10^{6}$ and $L_{n}$ is large as the nanoparticle fraction decrement $\left(\varphi_{1}-\varphi_{0}\right)$ in not smaller than $10^{-3}$ whereas $\mathrm{N}_{\mathrm{A}}$ is not greater than about 10 . Then, the exponents in equation (35) are small. By applying this approximation, the basic solution is found to be

$$
T_{b}=1-z, C_{b}=1-\mathrm{z} \text { and } \varphi_{b}=z \text {. }
$$

These results are identical with the results obtained by Kuznetsov and Nield (2010a) and Nield and Kuznetsov (2011).

\section{PERTURBATION SOLUTIONS}

We superimposed infinitesimal small perturbations on the basic state

$$
\begin{aligned}
& \mathbf{q}(u, v, w)=0+q^{\prime \prime}(u, v, w), T=(1-z)+T^{\prime \prime}, \\
& \mathrm{C}_{\mathrm{b}}=(1-z)+C^{\prime \prime}, \varphi=z+\varphi^{\prime \prime}, p=p_{b}+p^{\prime \prime} .
\end{aligned}
$$

Linearizing the Eqs. (8) - (12) by using the basic state (37) by neglecting nonlinear terms that are product of prime quantities and dropping the primes (") for convenience, we obtain

$$
\begin{gathered}
\nabla . \mathbf{q}=0, \\
\frac{1}{P_{r}} \frac{\partial \mathbf{q}}{\partial t}=-\nabla p+\nabla^{2} \mathbf{q}+\mathrm{R}_{\mathrm{D}} \mathrm{T} \hat{e}_{z}+\frac{R s}{L e} C \hat{e}_{z} \\
-\operatorname{Rn} \varphi \hat{e}_{z}+\sqrt{T a}\left(\mathbf{q} \times \hat{e}_{z}\right), \\
\frac{1}{\sigma} \frac{\partial \varphi}{\partial t}+w=\frac{1}{L n} \nabla^{2} \varphi+\frac{N_{A}}{L n} \nabla^{2} T, \\
\frac{\partial T}{\partial t}-w=\nabla^{2} T+\frac{N_{B}}{L n}\left(\frac{\partial T}{\partial z}-\frac{\partial \varphi}{\partial z}\right)
\end{gathered}
$$

$$
\begin{gathered}
-\frac{2 \mathrm{~N}_{A} N_{B}}{L n} \frac{\partial T}{\partial \mathrm{z}}+\mathrm{N}_{\mathrm{TC}} \nabla^{2} \mathrm{C} . \\
\frac{\partial C}{\partial t}-w=\frac{1}{L e} \nabla^{2} C+N_{C T} \nabla^{2} T .
\end{gathered}
$$

Boundary conditions for Eqs. (38) - (40) are

$$
w=0, T=0, \mathrm{C}=0, \varphi=0 \text { at } z=0.1 \text {. }
$$

The parameter $R m$ is not involved in Eqs. (38) (42), it is just a measure of the basic static pressure gradient.

The seven unknown's $u, v, w, p, T, C$ and $\varphi$ can be reduced to four by operating Eq. (39) with $e_{z}$.curl curl, which yields

$$
\begin{aligned}
& \frac{1}{P_{r}} \frac{\partial}{\partial t} \nabla^{2} w-\nabla^{4} w=R_{D} \nabla_{H}^{2} T+\frac{R s}{L e} \nabla_{H}^{2} C \\
& -R n \nabla_{H}^{2} \varphi-\sqrt{T a} \frac{\partial \zeta}{\partial z},
\end{aligned}
$$

where $\nabla_{H}^{2}=\frac{\partial}{\partial x^{2}}+\frac{\partial}{\partial y^{2}}$. is the two-dimensional Laplace operator on the horizontal plane and $\zeta=\hat{e}_{z}$.curlq is the z-component of vorticity.

Operating Eq. (39) by $\hat{e}_{z} . c u r l$, we get

$$
\left[\nabla^{2}-\frac{1}{P_{r}} \frac{\partial}{\partial t}\right] \zeta=\sqrt{T a} \frac{\partial w}{\partial z}
$$

Eliminating $\zeta$ from Eq. (44) by using Eq. (45), we get

$$
\begin{aligned}
& \frac{1}{P_{r}} \frac{\partial}{\partial t} \nabla^{2} w-\nabla^{4} w=R_{D} \nabla_{H}^{2} T+\frac{R s}{L e} \nabla_{H}^{2} C \\
& -R n \nabla_{H}^{2} \varphi+\left(\nabla^{2}-\frac{1}{P_{r}} \frac{\partial}{\partial t}\right)^{-1} T a \frac{\partial^{2} w}{\partial z^{2}} .
\end{aligned}
$$

\section{NORMAL MODES ANALYSIS} the form

Express the disturbances into normal modes of

$$
\begin{aligned}
& {[w, T, C, \varphi]=[W(z), \Theta(z), \Gamma(z), \Phi(z)]} \\
& \exp (i l x+i m y+\omega t),
\end{aligned}
$$


where $l, m$ are the wave numbers in the $x$ and $y$ direction, respectively, and $\omega$ is the growth rate of the disturbances.

Using Eq. (47) into Eqs. (46) and (40)-(42), we have an eigen value problem

$$
\begin{gathered}
{\left[\left(D^{2}-a^{2}\right)\left(D^{2}-a^{2}-\frac{\omega}{P_{r}}\right)^{2}+T a D^{2}\right] W} \\
-a^{2} R_{D}\left(D^{2}-a^{2}-\frac{\omega}{P_{r}}\right) \Theta \\
-\frac{R s}{L e} a^{2}\left(D^{2}-a^{2}-\frac{\omega}{P_{r}}\right) \Gamma \\
+a^{2} R n\left(D^{2}-a^{2}-\frac{\omega}{P_{r}}\right) \Phi=0, \\
W+N_{C T}\left(D^{2}-a^{2}\right) \Theta+ \\
\frac{1}{L e}\left(\left(D^{2}-a^{2}\right)-\omega\right) \Gamma=0, \\
W+\left(\frac{1}{L n}\left(D^{2}-a^{2}\right)-\omega\right) \Phi=0, \\
-\frac{\left.D^{2}+\frac{N_{B}}{L n} D^{2}-\frac{2 N_{A} N_{B}}{L n} D-a^{2}-\omega\right) \Theta}{L n} D+N_{T C}\left(D^{2}-a^{2}\right) \Gamma=0, \\
W, \Gamma=0, \Theta=0, \Phi=0 \mathrm{at} \quad \mathrm{Z}=0
\end{gathered}
$$

and

$$
W=0, D^{2} W=0, \Gamma=0, \Theta=0, \Phi=0 \text { at } z=1 \text {. }
$$

where $D=\frac{d}{d z}$ and $a^{2}=l^{2}+m^{2}$ is the dimensionless horizontal wave number.

\section{DISPERSION RELATION}

Consider the solutions of the form

$$
\begin{aligned}
& W=W_{0} \sin (\pi z), \Theta=\Theta_{0} \sin (\pi z), \\
& \Gamma=\Gamma_{0} \sin (\pi z), \Phi=\Phi_{0} \sin (\pi z) .
\end{aligned}
$$

Using (54) in the system of Eqs. (48) - (51) and integrating each equation from $z=0$ to $z=1$, we obtain the following matrix equations

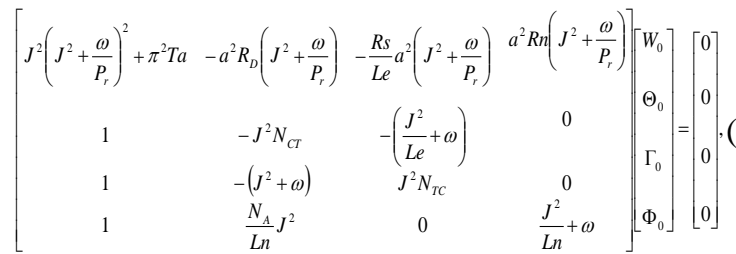

where $J^{2}=\pi^{2}+a^{2}$ is the total wave number.

The linear system (55) has a non-trivial solution if and only if

$$
R_{D}=\frac{1}{J^{2}+\omega L e-N_{T C} J^{2} L e}\left\{\begin{array}{l}
\frac{P_{r}}{a^{2}\left(J^{2} P_{r}+\omega\right)}\left[\begin{array}{l}
\left(J^{2}\left(\frac{J^{2} P_{r}+\omega}{P_{r}}\right)^{2}+\pi^{2} T a\right) \times \\
\left(\left(J^{2}+\omega\right)\left(J^{2}+\omega L e\right)-L e N_{T C} N_{C T} J^{4}\right)
\end{array}\right] \\
+\frac{R s}{a^{2}}\left[N_{C T} J^{2}-\left(J^{2}+\omega\right)\right]+\left(\frac{R n L n}{J^{2}+\omega L n}\right)\left(\frac{J^{2} P_{r}+\omega}{P_{r}}\right) \times x \\
{\left[\begin{array}{l}
J^{4} N_{T C} N_{C T}+\left(J^{2}+\omega\right)\left(J^{2}+\omega L e\right)- \\
\frac{N_{\mathrm{A}}}{\mathrm{Ln}} J^{2}\left(J^{2} N_{\mathrm{TC}}+J^{2}+\omega L e\right)
\end{array}\right]}
\end{array}\right\}
$$

Eq. (56) is the required dispersion relation describing for the effect of thermo-solutal Lewis number, modified Taylor number, thermo-nanofluid Lewis number, kinematic visco-elasticity parameter, solutal Rayleigh Number, nanoparticle Rayleigh number, modified diffusivity ratio, Soret and Dufour parameter on double diffusive convection in a layer of nanofluid.

\section{THE STATIONARY CONVECTION}

For stationary convection, we put $\omega=0$ in equation (56) reduces it to

$R_{\mathrm{D}}=\frac{1}{\left(1-N_{T C} L e\right) a^{2}} \times$

$\left\{\begin{array}{l}\left(\pi^{2}+a^{2}\right)\left(\left(\pi^{2}+a^{2}\right)^{3}+\pi^{2} T a\right)\left(1-L e N_{T C} N_{C T}\right)+R s\left(N_{C T}-1\right) \\ +a^{2} R n\left(\pi^{2}+a^{2}\right)\left[L n+L n N_{C T} N_{T C}-N_{A}\left(1+N_{\mathrm{TC}}\right)\right]\end{array}\right\}$.

Eq. (57) expresses the thermal Darcy-Rayleigh number as a function of the dimensionless resultant wave number $a$ and the parameters Ta, $N_{T C}, N_{C T}, R s, L n, R n, L e, N_{A}$. Eq. (57) is identical to that obtained by Kuznetsov and Nield $\left(2010^{\mathrm{a}}\right)$, and Chand and Rana (2012b). Also in Eq. (57) the particle increment parameter $N_{B}$ does not appear and the diffusivity ratio parameter $N_{A}$ appears only in association with the nanoparticle Rayleigh number $R n$. This implies that the nanofluid crossdiffusion terms approach to be dominated by the regular cross-diffusion term.

In the absence of Dufour and Soret parameters $N_{T C}$ and $N_{C T}$ equation (57) reduces to 


$$
\begin{aligned}
& R_{D}=\frac{\left(\pi^{2}+a^{2}\right)\left(\left(\pi^{2}+a^{2}\right)^{3}+\pi^{2} T a\right)}{a^{2}} \\
& -\frac{R s}{a^{2}}-\left(\pi^{2}+a^{2}\right)\left(\operatorname{Ln}+N_{A}\right) R n,
\end{aligned}
$$

which is identical with the equation derived by Rana et al. (2014).

In the absence of rotation, equation (58) reduces to

$$
\begin{aligned}
& R_{D}=\frac{\left(\pi^{2}+a^{2}\right)^{4}}{a^{2}}-\frac{R s}{a^{2}} \\
& -\left(\pi^{2}+a^{2}\right)\left(L n+N_{A}\right) R n,
\end{aligned}
$$

which is same with the result derived by Kuznetsov and Nield (2010a).

The result given in equation (59) is a good agreement with the result derived by Kuznetsov and Nield (2010a) and Chand and Rana (2012b) in the absence of the Dufour, Soret and stable solute gradient parameters $N_{T C}, N_{C T}$ and Rs respectively. Eq. (57) is analyzed numerically to depict the stability characteristics with respect to rotation.

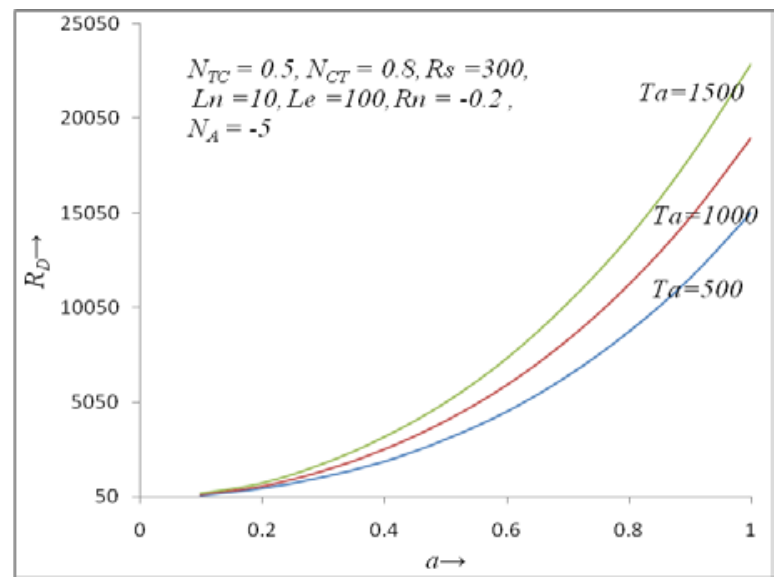

Figure 2. Variation of Darcy-Rayleigh number $R_{D}$ with respect to wave number $a$ for $T_{a}=500,1000$, 1500.

It follows from the above graph that as Taylor number $\mathrm{Ta}$ increases then Darcy-Rayleigh number $R_{D}$ also increases. This means that a nanofluid, in rotation, is stable with respect to the onset of stationary convection when both the boundaries are free. This result is in good agreement with the result obtained by Chand and Rana(2012b).

\section{CONCLUSIONS}

The effect of rotation on the onset doublediffusive convection in a layer of Nanofluid is investigated by using a linear stability analysis and Galerkin-type weighted method. The solutal Rayleigh Number, thermo-nanofluid Lewis number and Soret parameter have stabilizing effects on the stationary convection which are in good agreement with the results as discussed by Kuznetsov and Nield (2010a) and Rana et al. (2014). The thermo-solutal Lewis number and Dufour parameter has destabilizing effects on the stationary convection of the system which are identical with the results as derived by Kuznetsov and Nield [12] and Chand and Rana (2012b). Rotation has stabilizing effect on the stationary convection as shown in figure 2 which is in good agreement with the result derived by and Rana (2013). Rotation plays important role in the thermal instability of fluid layer and has applications in rotating machineries such as petroleum industry, biomechanics etc.

\section{REFERENCES}

Alloui, Z., Vasseur, P., and Reggio, M., 2011, Natural Convection of Nanofluids in a Shallow Cavity Heated from Below, International Journal of Thermal Sciences, Vol. 50, pp. 385-393.

Brakke, M. K, 1955, Zone Electrophoresis of Dyes, Proteins and Viruses in Density Gradient Columns of Sucrose Solutions, Archives of Biochemistry and Biophysics, Vol. 55, pp.175-190.

Buongiorno, J., 2006, Convective Transport in Nanofluids, ASME Journal of Heat Transfer, Vol. 128, pp. 240-250.

Chand, R., and Rana, G. C., 2012, On the Onset of Thermal Convection in Rotating Nanofluid Layer Saturating a Darcy-Brinkman Porous Medium, International Journal of Heat and Mass Transfer, Vol. 55, pp. 5417-5424.

Chand, R., and Rana, G. C., 2012, Dufour and Soret Effects on the Thermosolutal Instability of Rivlin-Ericksen Elastic-Viscous Fluid in Porous Medium, Zeitschrift fur Naturforschung, Vol. 6, pp. 685-691.

Chandrasekhar, S., 1961, Hydrodynamic and Hydromagnetic Stability, Dover Publication, New York.

Choi, S., 1995, Enhancing Thermal Conductivity of F luids with Nanoparticles, in: D. A., Siginer and H. P., Wang (Eds), Developments and Applications of Non-Newtonian Flows, ASME FED, 231/MD, Vol. 66, pp. 99-105.

Kuznetsov, A. V., and Nield, D. A., 2010, Thermal Instability in a Porous Medium Layer Saturated by a Nanofluid: Brinkman Model, Transport in Porous Media, Vol. 81, pp. 409-422.

Kuznetsov, A. V., and Nield, D. A., 2010, The Onset of Double-Diffusive Nanofluid Convection in a Layer of Saturated Porous Medium, Transport in Porous Media, Vol. 85, pp. 941-951.

Nield, D. A., and Kuznetsov, A. V., 2009, Thermal Instability in a Porous Medium Layer 
Saturated by a Nanofluid, International Journal of Heat Transfer, Vol. 52, pp. 5796-580.

Nield, D. A., and Kuznetsov, A. V., 2011, The Onset of Double-Diffusive Convection in a Nanofluid Layer, International Journal of Heat and Fluid Flow, Vol. 32, pp. 771-776.

Rana, G. C., 2013, Thermosolutal Convection in Walters' (Model B') Rotating Fluid Permeated with Suspended Particles and Variable Gravity Field in Porous Medium in Hydromagnetics, Journal of Applied Fluid Mechanics, Vol. 6, No. 1, pp. 87-94.

Rana, G. C., Kango, S. K., and Kumar, S., 2012, Effect of Rotation on the Onset of Convection in Walters' (Model B') Fluid Heated from Below in a Darcy-Brinkman Porous Medium, Journal of Porous Media, Vol. 15, pp. 1149-1153.

Rana, G. C., Thakur, R. C., and Kango, S. K., 2014, On the Onset of Thermosolutal Instability in a Layer of an Elastico-Viscous Nanofluid in Porous Medium, FME-Transactions, Vol. 42, pp. 1-9.

Sharma V., Kishore, K., and Rana, G. C., 2001, The Instability of Streaming Rivlin-Ericksen Fluids in Porous Medium, Studia Geotechnical et Mechanica, Vol. XIII, pp. 83-93.

Stomel, H., and Fedorov, K. N., 1967, Small Scale Structure in Temperature and Salinity Near Timor and Mindanao, Tellus, Vol. 19, pp. 306-325

Turner, J. S., 1974, Double-Diffusive Phenomena, Annual Review of Fluid Mechanics, Vol. 6, pp. 37-56.

Tzou, D. Y., 2008, Thermal Instability of Nanofluids in Natural Convection, International Journal of Heat and Mass Transfer, Vol. 5, pp. 2967-2979. 